Int. J. Dev. Biol. 54: 1089-1098 (2010)

doi: $10.1387 / \mathrm{ijdb} .103070 \mathrm{cg}$

\title{
Hematopoietic stem cell development in the placenta
}

\author{
CHRISTOS GEKAS, KATRIN E. RHODES, BEN VAN HANDEL, AKANKSHA CHHABRA, \\ MASAYA UENO and HANNA K.A. MIKKOLA* \\ University of California Los Angeles, Los Angeles, CA, USA
}

\begin{abstract}
The placenta is a highly vascularized organ that mediates fetal-maternal exchange during pregnancy and is thereby vital for the survival and growth of the developing embryo. In addition to having this well-established role in supporting pregnancy, the placenta was recently shown to function as a hematopoietic organ. The placenta is unique among other fetal hematopoietic organs, as it is capable of both generating multipotential hematopoietic cells de novo and establishing a major hematopoietic stem cell (HSC) pool in the conceptus, while protecting HSCs from premature differentiation. The mouse placenta contains two distinct vascular regions that support hematopoiesis: the large vessels in the chorionic plate where HSCs/progenitors are thought to emerge and the labyrinth vasculature where nascent HSCs/progenitors may colonize for expansion and possible functional maturation. Defining how this cytokine- and growth factor rich organ supports HSC generation, maturation and expansion may ultimately help to establish culture protocols for HSC expansion or de novo generation from pluripotent cells.
\end{abstract}

KEY WORDS: placenta, hematopoietic stem cell, niche, allantois, hemogenic endothelium

\section{Introduction}

Continuous blood cell production throughout the lifetime of an individual is ensured by hematopoietic stem cells (HSCs), which are rare bone marrow cells that possess extensive self-renewal capacity and ability to differentiate to all blood cell lineages. Due to the unique properties of HSCs, they can regenerate the entire hematopoietic system of a recipient upon transplantation and thereby provide a cure for inherited and acquired blood diseases. As such, HSCs are of substantial therapeutic interest. However, the limited availability of human leukocyte antigen (HLA) matched bone marrow donors and the relatively low yield of HSCs in more accessible sources, such as cord blood, limits the number of patients that can be treated. Therefore, a great need exists for improving culture methods that would facilitate HSC expansion, or de novo HSC generation from pluripotent cells such as human embryonic stem cells (ES) or induced pluripotent stem (iPS) cells. To succeed in these endeavors, major emphasis has been put on understanding HSC development and the microenvironmental niches that support this process. As a result, a revised model for HSC development is emerging that includes a greater understanding of both the anatomical sites and cellular niches where HSCs develop as well as the cell of origin for HSCs.

HSCs are formed only within a limited time window during embryogenesis, after which the HSC pool is maintained by self- renewing cell divisions. Unlike post-natal hematopoiesis that is confined to the bone marrow, fetal hematopoiesis occurs in multiple different anatomical sites in a temporally defined manner (Mikkola and Orkin, 2006). The utilization of multiple hematopoietic sites during fetal development facilitates both the rapid generation of the first differentiated blood cells that the embryo needs for survival and growth, as well as the establishment of a large pool of undifferentiated HSCs that are required for postnatal hematopoiesis. The first hematopoietic activity is observed in the extra-embryonic yolk sac, which generates large cohorts of uni- and oligolineage progenitors that are non-self-renewing and restricted to erythroid and myeloid fates, whereas the truly multipotential, self-renewing HSCs are generated later. These longterm reconstituting HSCs emerge in multiple anatomical sites, and once generated, convene in the fetal liver for expansion and differentiation prior to their colonization of the bone marrow. During this journey, the developing HSCs are exposed to distinct extracellular cues that promote their generation, expansion, and functional maturation to acquire the adult HSC phenotype and functional properties. Until recently, the aorta-gonad mesonephros (AGM) region and adjacent vitelline and umbilical arteries

Abbreviations used in this paper:AGM, aorta-gonad mesonephros; CFU, colony forming unit; HSC, hematopoietic stem cell.

\footnotetext{
*Address correspondence to: Hanna Mikkola. 621 Charles E Young Drive South, LS 2204, University of California Los Angeles, Los Angeles, CA, 90095, USA. Fax: +1-310-206-5553. e-mail: hmikkola@mcdb.ucla.edu
} 
were considered to be the main source of HSCs during embryogenesis (Cumano et al., 1996; de Bruijn et al., 2000; Godin et al., 1999; Medvinsky and Dzierzak, 1996; Muller et al., 1994). However, due to the low number of transplantable HSCs found in the AGM, it was questioned whether this scarce population could be the sole contributor to the substantial amount of HSCs found in the fetal liver. As the fetal liver is incapable of de novo generation of HSCs and hematopoietic progenitors, it was hypothesized that other embryonic sites such as the yolk sac, or yet unknown sites, may contribute to the seeding of the HSCs to the fetal liver (Kumaravelu et al., 2002). Studies in recent years have revealed that the placenta also harbors a major HSC pool, which may in part account for the "missing HSCs" from the equation. Notably, the placenta appears to be unique among the other fetal hematopoietic organs, as it not only has the capacity for de novo hematopoiesis but it also accrues a large reservoir of HSCs and protects them from signals that promote immediate differentiation. The placenta is a highly vascularized organ that is rich in cytokines and growth factors that likely contribute to the hematopoietic microenvironment (Cross, 2005). Hence, the discovery of the placenta as a major fetal HSC niche has opened new avenues of research focused on defining the unique microenvironmental cues that support HSC development, and offered access to larger numbers of developing HSCs from both mice and humans (Barcena etal., 2009a; Barcena etal., 2009b; Gekas etal., 2005; Ottersbach and Dzierzak, 2005; Robin et al., 2009). Here, we review how the placenta was unveiled as a fetal hematopoietic organ and discuss its unique function as a specialized niche for HSC development.

\section{Discovery of hematopoietic stem- and progenitor cell activity in the mouse placenta}

The placenta has not traditionally been regarded as a hematopoietic organ, although the first report suggesting a possible role for the placenta in blood development was published already in 1961 (Till and McCulloch, 1961). That study discovered that transplantation of mouse placental tissue into irradiated recipient mice leads to the formation of hematopoietic colonies in the spleen (CFU-S, colony forming unit spleen; Table 1). Although it is now known that the hematopoietic cells that form CFU-S are not necessarily true long-term repopulating HSCs (LTR-HSCs), this early study evidenced that the placenta harbors a robust population of clonogenic hematopoietic cells with high proliferative potential. Another study published in 1977 provided compelling evidence that the placenta harbors HSCs, as placental tissue exhibited the capacity to rescue the anemic phenotype of W/WV recipient mice upon transplantation (Dancis et al., 1977) (Table 1). Interestingly, separation of placenta from the embryonic circulation did not abolish hematopoietic reconstitution ability implying that accumulation of HSCs in the placenta was not dependent on the continuous supply of blood to the placenta. Notably, these authors had already previously reported the presence of immunologically competent fetal hematopoietic cells in the placenta similar to the spleen, as graft-versus-host disease ensued after transplantation of placenta from C57 mice into BALB/C mice (Dancis et al., 1962). Furthermore, another study showed that the placenta is an early reservoir for B cell precursors, as plaqueforming assays documented robust B-cell potential in the midgestation placenta prior to the fetal liver (Melchers, 1979). In
TABLE 1

\section{HISTORICAL PERSPECTIVE ON THE DISCOVERY OF HSCs IN THE PLACENTA}

\begin{tabular}{|c|c|c|c|}
\hline Discovery & Methods & Authors & Year \\
\hline $\begin{array}{l}\text { Transplantable CFU-S10 in E11-12 } \\
\text { mouse placenta }\end{array}$ & $\begin{array}{l}\text { Transplantation into lethally } \\
\text { irradiated mice }\end{array}$ & Till and McCulloch & 1961 \\
\hline $\begin{array}{l}\text { Graft-versus-host disease upon } \\
\text { transplantation of placental cells into } \\
\text { allogeneic recipients suggests } \\
\text { presence of immunologically } \\
\text { competent hematopoietic cells. }\end{array}$ & $\begin{array}{l}\text { Transplantation into lethally } \\
\text { irradiated allogeneic mice }\end{array}$ & Dancis et al. & 1962 \\
\hline $\begin{array}{l}\text { Sustained rescue of anemia of W/Wv } \\
\text { mice after transplantation of E15, but } \\
\text { not E18-19, placenta cells, suggested } \\
\text { transient presence of HSCs }\end{array}$ & $\begin{array}{l}\text { Transplantation into sub- } \\
\text { lethally irradiated W/Wv } \\
\text { mice }\end{array}$ & Dancis et al. & 1977 \\
\hline $\begin{array}{l}\text { Plaque-forming B-cell progenitors } \\
\text { present in midgestation placenta prior } \\
\text { to fetal liver }\end{array}$ & $\begin{array}{l}\text { In-vitro stroma co-culture } \\
\text { assays }\end{array}$ & Melchers & 1979 \\
\hline $\begin{array}{l}\text { Multipotent hematopoietic potential } \\
\text { suggestive of HSCs in the avian } \\
\text { allantois }\end{array}$ & $\begin{array}{l}\text { Chick-to-quail grafting } \\
\text { experiments }\end{array}$ & Caprioli et al. & $\begin{array}{l}1998, \\
2001\end{array}$ \\
\hline $\begin{array}{l}\text { Multipotent hematopoietic progenitors } \\
\text { of fetal origin in mouse placenta prior } \\
\text { to fetal liver (E9.0 onwards) }\end{array}$ & $\begin{array}{l}\text { In vitro clonogenic assays } \\
\text { using GFP+ fetal cells }\end{array}$ & Alvarez-Silva et al. & 2003 \\
\hline $\begin{array}{l}\text { Placenta is a major niche of HSCs } \\
\text { during midgestation while protecting } \\
\text { HSCs from differentiation }\end{array}$ & $\begin{array}{l}\text { Serial transplantations into } \\
\text { lethally irradiated mice, in } \\
\text { vitro clonogenic assays }\end{array}$ & Gekas et al. & 2005 \\
\hline $\begin{array}{l}\text { HSCs were found in placental } \\
\text { labyrinth }\end{array}$ & $\begin{array}{l}\text { Localization of } \mathrm{HS} / \mathrm{PCs} \text { in } \\
\text { Sca1-GFP model, } \\
\text { transplantation into lethally } \\
\text { irradiated mice }\end{array}$ & $\begin{array}{l}\text { Ottersbach and } \\
\text { Dzierzak }\end{array}$ & 2005 \\
\hline $\begin{array}{l}\text { Allantoic and chorionic mesoderm are } \\
\text { capable of generating hematopoietic } \\
\text { progenitors de novo }\end{array}$ & $\begin{array}{l}\text { Localization of hemogenic } \\
\text { precursors in Runx1-LacZ } \\
\text { embryos, in vitro stroma co- } \\
\text { culture assays }\end{array}$ & Zeigler et al. & 2006 \\
\hline
\end{tabular}

spite of these intriguing findings that hinted at an important role for the placenta in fetal hematopoiesis, further work on the placenta as a hematopoietic organ did not commence until decades later.

Interest in the placenta as a hematopoietic organ was revived after work by Dieterlen-Liévre, Jaffredo and colleagues showed that the avian allantois harbors multipotential hematopoietic precursors prior to the onset of circulation (Caprioli et al., 1998; Caprioli et al., 2001) (Table 1). The avian allantois is a mesodermal appendage derived from the primitive streak, and has both a similar developmental origin and function in nutrient and gas exchange as the placenta in eutherial mammals. The discovery that the avian allantois possesses de novo hematopoietic activity brought up the question whether the mammalian placenta is also capable of generating HSCs.

Studies by Dieterlen-Liévre and colleagues indeed revealed that the mouse placenta harbors a major pool of multipotential hematopoietic progenitors that are of fetal origin (Alvarez-Silva et al., 2003) (Table 1). To verify that the placental hematopoietic progenitors were not maternal cells that were circulating through the placenta, GFP-reporter transgenic male mice were bred with non-transgenic females, allowing distinction between fetal $\left(\mathrm{GFP}^{+}\right)$ and maternal (GFP') cells. Methylcellulose colony forming assays showed that the placenta harbors clonogenic progenitors by the $20 \mathrm{sp}$ (somite pair) stage (E9.0), shortly after the yolk sac (15sp) and the caudal half of the embryo (18sp), which contains the paraaortic splanchopleura ( $p S P$ ) that gives rise to the AGM region (Table 1). In contrast, colony-forming units (CFUs) were not found in the fetal liver until later in development. Furthermore, the distribution of CFU types in the placenta and the fetal liver were 
different: the frequency of the multipotential, as compared to committed, unilineage, progenitors in the placenta was much higher than in the fetal liver, implying that the hematopoietic programs in these organs and the microenvironments that support these programs are different from one another (Alvarez-Silva et al., 2003).

However, progenitor activity does not necessarily correlate with the presence of true HSCs. As indicated before, lineagerestricted, transient progenitors develop during embryogenesis before the appearance of the first definitive HSCs. Therefore, it was critical to assess HSC activity in vivo by stringent transplantation assays. Two independent studies verified that the midgestation placenta harbors true adult-reconstituting HSCs that can generate all blood cell lineages and self-renew through serial transplantations (Gekas et al., 2005; Ottersbach and Dzierzak, 2005) (Table 1). To verify fetal origin of placental hematopoietic cells, Ottersbach and Dzierzak generated transgenic fetuses using male mice expressing human beta-globin from a ubiquitous promoter or GFP from the Sca1 (Ly-6A) promoter. In the other study, Gekas and colleagues bred embryos that were heterozygous for the two different alleles of the panhematopoietic marker CD45 (CD45.1 and CD45.2), distinguishing them from maternal cells that only expressed the CD45.2 allele. These studies showed that the placenta harbors adult reconstituting $\mathrm{HSCs}$ at the same developmental stage or slightly later than the AGM (E10.5-11.0, 33-40sp). At that stage, no HSCs were found in circulating blood or the fetal liver rudiment, supporting the hypothesis that placental HSCs could at least in part be generated de novo in the placenta (Fig. 1A) (Gekas et al. 2005). Hematopoietic reconstitution by placental HSCs was multilineage and sustained through serial transplantations, as shown by flow cytometry analysis of blood and bone marrow from primary and secondary recipients. Furthermore, functional analysis of prospectively isolated sub-fractions of placental cells revealed that at E12.5, placental HSCs and multipotential progenitors possess the classical CD34 ${ }^{\text {med }} \mathrm{c}-\mathrm{kit}^{\text {hi }}$ fetal HSC surface phenotype, similar to the fetal liver HSCs (Fig. 2A) (Gekas et al. 2005). Although these studies were not able to unequivocally reveal the origin of placental HSCs, they were the first to describe the placenta as a hematopoietic organ that harbors classical HSCs with all the essential properties of long-term reconstituting HSCs.

\section{Kinetics of HSCs and progenitors in fetal hematopoi- etic organs}

The potential impact of the discovery of the placenta as a novel source of HSCs was further strengthened by the finding that the number of HSCs in the placenta surpassed that in the AGM and yolk sac, the two known sites with de novo hematopoietic activity. In contrast to the AGM and the yolk sac where the number of HSCs remains low, the HSC pool in the placenta increases drastically and, at its peak at E12.5-13.5, the placenta harbors 15fold more HSCs than the AGM or the yolk sac (Gekas et al. 2005) (Fig. 1A). The dramatic increase in HSCs occurring in the placenta between E11.5 and E12.5 ( 20x) is comparable to that of the fetal liver $(\sim 28 \mathrm{x})$, and not seen in any other fetal hematopoietic organ. Interestingly, although the number of reconstituting units in the fetal liver was higher than in the placenta at E12.5, the average reconstitution level from placental HSCs vs. fetal liver HSCs was higher, implying that the placental HSCs at this stage are more potent (Gekas et al. 2005). After E13.5, the placental HSC pool decreases greatly and a concomitant increase of HSC number can be seen in the fetal liver; at this stage the average reconstitution ability of fetal liver HSCs is also higher. These data suggested that the placental HSCs might seed the fetal liver, which is directly downstream of the placenta in fetal circulation.

The analysis of clonogenic progenitor frequencies throughout gestation by Gekas and colleagues provided further insight into the distinct roles of the different hematopoietic tissues. While CFU-Cs increased in the fetal liver between E11.5 to E12.5 $(\sim 13 x)$, CFU-Cs in the placenta only increased two-fold despite the much greater increase in transplantable HSCs during this time (Fig. 1A, B). This provided functional evidence that the placental microenvironment is geared towards supporting the expansion
A

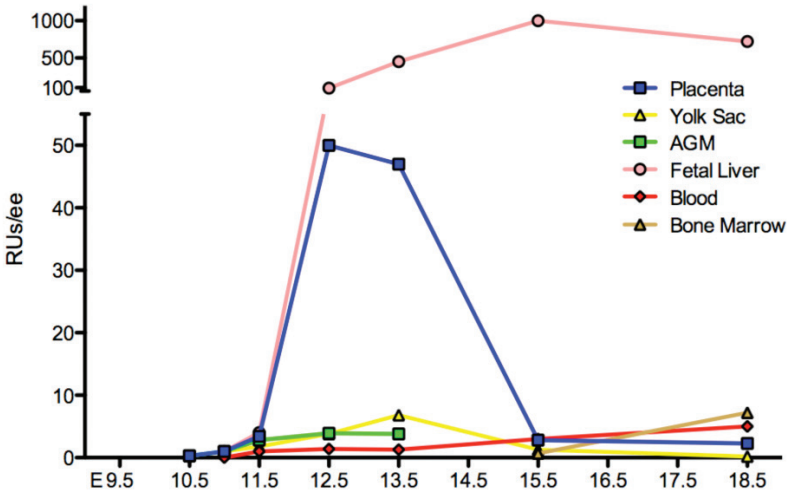

B

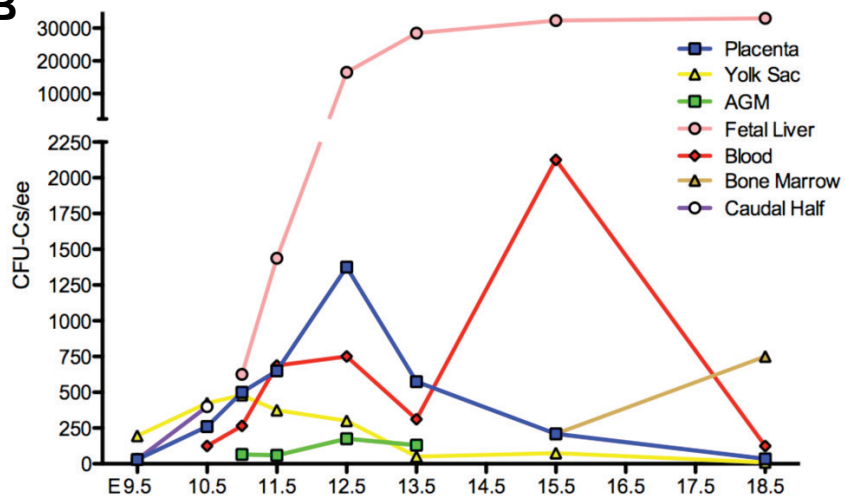

Fig. 1. Kinetics of long-term reconstituting HSCs and clonogenic progenitors in the embryo and extraembryonic hematopoietic tissues. (A) The graph depicts the number of HSCs with long-term reconstitution capability per tissue during development. The midgestation placenta harbors a large pool of HSCs, which diminishes towards the end of gestation while the fetal liver HSC pool is expanding. (B) The diagram shows numbers of clonogenic progenitors (CFU-C) per embryo equivalent of tissue. Despite the major increase of HSCs in placenta between E11.5-12.5 (Fig. 1A), the number of CFU-Cs increases only modestly. In contrast, a substantial expansion of CFU-Cs can be noted in fetal liver throughout gestation. Note that presence of circulating progenitors in the bloodstream does not correlate with hematopoietic kinetics in placenta. RU, reconstituting unit; ee, embryo equivalent, CFU-C, colony forming unit-colony. Caudal half (E10.5) includes the dorsal aorta and fetal liver rudiment. (Gekas et al. 2005) 
and/or maturation of HSCs without promoting their concomitant differentiation into myeloerythroid progenitors (Fig. 1B). In contrast, the fetal liver produces major populations of CD34 ${ }^{-} \mathrm{c}-\mathrm{kit}^{\mathrm{hi}}$ erythro-megakaryocytic progenitor cells and proerythroblasts, indicative of ongoing definitive erythropoiesis (Fig. 2A and B). In contrast, the only substantial erythroid populations in the midgestation placenta consist of primitive erythroblasts that are circulating through the placenta, as well as enucleated maternal red blood cells (Fig. 2B). These data implicate inherent differences between fetal liver and placental HSC/progenitor populations and hematopoietic microenvironments, where the fetal liver, but not the placenta, promotes active definitive erythropoiesis during midgestation. These results are in accordance with the study from Alvarez-Silva et al., which showed that the placenta specifically harbors multipotential, high-proliferative potential progenitors that can be serially replated whereas the fetal liver is rich in unilineage progenitors (Alvarez-Silva et al., 2003). These

A
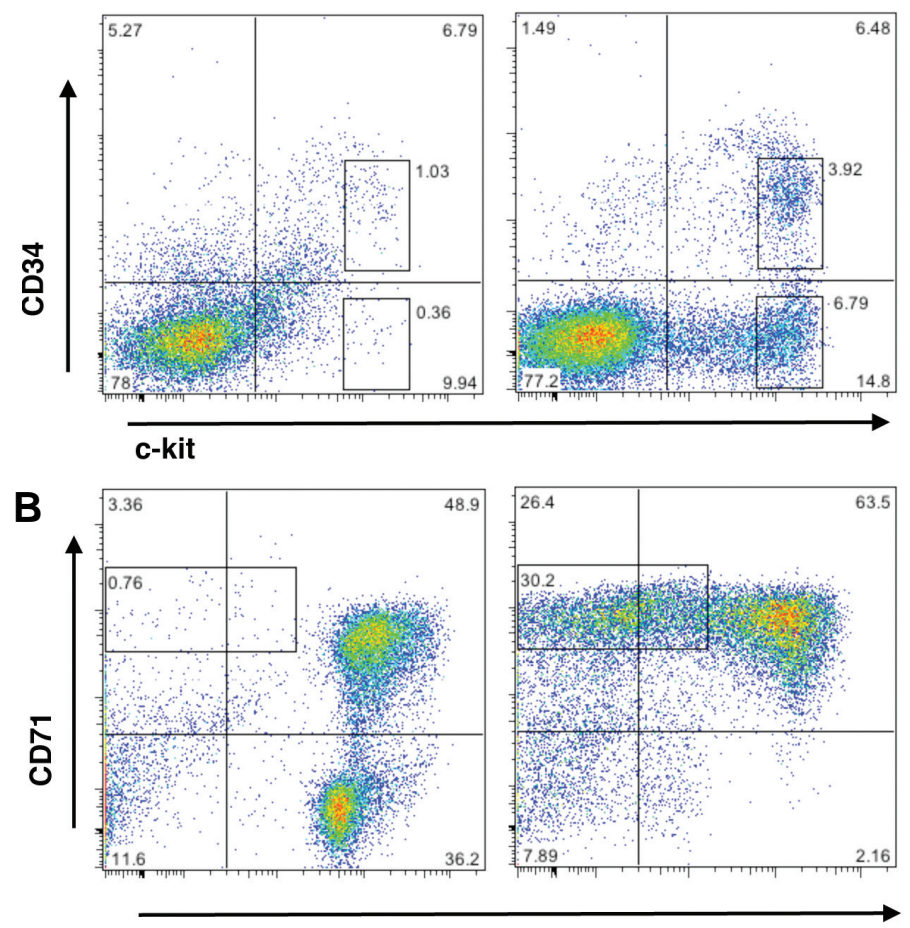

Ter119

Fig. 2. Distribution of placental and fetal liver HSCs/multipotential progenitors and committed progenitors. (A) Surface phenotype analysis of HSCs and progenitors in E13.5 placenta and fetal liver by flow cytometry for expression of CD34 and c-kit. The $\mathrm{CD}_{34}{ }^{+} \mathrm{ckithi}$ fraction (upper boxed region) harbors HSCs and multipotential progenitors, whereas the $c-k$ it $^{+}$fraction (lower boxed region) harbors progenitors that are committed to erythroid and megakaryocytic lineages. The fetal liver contains a much higher proportion of the lineage-committed progenitors than the placenta. (B) Distribution of erythroid precursors in the placenta and the fetal liver documents an abundance of $C D 71^{+}$Ter117proerythroblasts (boxed region), indicative of active definitive erythropoiesis, in the liver but not the placenta. The CD71+Ter119+ cells represent either primitive or definitive erythroblasts that contain a nucleus, whereas the CD71-Ter119+ cells represent enucleated fetal or maternal (placenta only) erythrocytes. studies pinpointed the uniqueness of the placental microenvironment as a niche that facilitates the establishment of a large pool of adult-type HSCs that are not directed towards lineage differentiation during their residence in the placenta.

\section{De novo hematopoiesis in the placenta}

In order to define the origin of placental HSCs and the niches that support their development, it is essential to understand how the placenta develops (Rossant and Cross, 2001). The placenta is an extra-embryonic organ that forms from trophoectoderm and mesodermal tissues. The two mesodermal components that form the placenta are the chorionic mesoderm, which lines the exocoelomic cavity, and the allantois, which grows from the posterior primitive streak of the epiblast into the exocoelomic cavity (Fig. 3A). The allantoic bud grows toward the ectoplacental cone and fuses with the chorionic mesoderm by E8.5 of mouse gestation (5sp stage), coinciding with the initiation of heartbeat and the onset of circulation in the embryo. The proximal part of the allantoic mesoderm becomes the umbilical cord that connects the placenta to the embryo, whereas the distal mesoderm that has fused with the chorionic mesoderm will both form the chorioallantoic mesenchyme in the chorionic plate and start to invade the trophoblast layer to establish the fetal vascular compartment of the placental labyrinth (Fig. 3B, C, D). After midgestation, two regions of fetal vasculature can be distinguished in the placenta: the chorioallantoic vessels and the labyrinth vessels.

The chorioallantoic vasculature is comprised of large vessels that are surrounded by mesenchyme and directly connect the placenta to the dorsal aorta and fetal liver through the umbilical cord vessels (Fig. 3C, D). This region of the placenta is primarily of mesodermal origin, although during midgestation it harbors unique tubular structures derived from ectoplacental endoderm (Fig. 4). Later during development, these endodermal cells form a cavity between chorioallantoic mesenchyme and the labyrinth known as the Crypt of Duval (Duval, 1891; Ogura etal., 1998). The labyrinth is composed of an intricate fetal vascular network that is intertwined with the trophoblast-lined maternal blood spaces (Fig. 3D). This region directly mediates fetal-maternal exchange of gases, waste products and nutrients (Fig. 3D). Next to the labyrinth layer is the spongiotrophoblast layer, or junctional zone, which provides both structural support for the placenta and a gateway for the maternal spiral arteries that bring the maternal blood to the placenta. Giant cells, which are large, multinucleated trophoblasts, form a thin layer between the spongiotrophoblasts and the maternal decidua. Hence, most of the placental tissue is of fetal origin, except for the maternal blood cells that circulate in the trophoblast spaces.

As all blood is derived from mesoderm, the chorionic and allantoic mesoderm are the candidate tissues of HSC origin in the placenta. To define whether the allantoic and/or chorionic mesoderm have a capacity for de novo hematopoiesis, these mesodermal tissues were isolated prior to chorioallantoic fusion and circulation and assayed for hematopoietic progenitor potential. Earlier studies had shown that the pre-circulation allantois does not harbor hematopoietic progenitors that form colonies in standard methylcellulose progenitor assays (Downs etal., 1998; Palis et al., 1999). Therefore, to assess whether the placenta contains the precursors that can generate hematopoietic stem/progenitor 
cells, hematopoietic potential had to be assessed by stimulating the cells from the tissues using in vitroco-culture on OP-9 stroma followed by methylcellulose culture, or by using an explant culture where hemogenic precursors remain in contact with their neighboring cells (Corbel et al., 2007; Zeigler et al., 2006)(Table 1). These studies revealed that both the chorionic and allantoic mesoderm are capable of generating myeloerythroid colonies, suggesting that the mesodermal tissues destined to become the placenta have innate potential to generate hematopoietic precursors. Furthermore, an earlier study in mice had shown that an ectopically transplanted murine allantois can contribute to the dorsal aorta and surrounding tissues (Downs and Harmann, 1997). Although hematopoietic potential of the transplanted allantoic mesoderm was not addressed in this study, these findings imply a similar developmental potential of the allantoic mesoderm as the lateral plate mesoderm in the embryo proper.

Verification of the sites of HSC emergence in the more developed embryo and placenta has been hindered by the fact that definitive HSCs do not emerge until a few days after the onset of heartbeat. Once circulation is established, the origin of HSCs and progenitors found within a tissue is difficult to prove. This issue was overcome using Ncx1 knockout embryos that lack heartbeat and circulation (Rhodes et al., 2008). These embryos are defective for the sodium calcium exchange pump 1 (Ncx1), which abolishes the initiation of heartbeat and thus prevents circulation (Koushik et al., 2001; Lux et al., 2008). As Ncx1/- embryos die by $\mathrm{E} 10.5$, it is not possible to functionally verify HSC potential by in vivo transplantation assays due to the immaturity of nascent HSCs, which precludes engraftment and reconstitution of adult bone marrow. Nevertheless, these studies showed that placental tissue, alike AGM and yolk sac, from E9.5 Ncx1/- embryos has robust multilineage myelo-lymphoid hematopoietic potential when cultured on supportive stroma (Rhodes et al., 2008). Specifically, the ability to generate myeloerythroid as well as B- and T- lymphoid cells indicates that placental hematopoietic cells have the differentiation potential characteristic of HSCs that is distinct from earlier, transient progenitor populations in the yolk sac, which solely generate myeloerythroid progeny. Taken together these studies implied that the placenta has the capacity to initiate multilineage hematopoiesis de novo.

\section{Placental hematopoietic niches}

To understand what regions of the placenta support hematopoiesis, localization of developing hematopoietic stem- and progenitor cells in the placenta was mapped using markers that are expressed in nascent HS/PCs (hematopoietic stem- and progenitor cells). To that end, Runx1-LacZ reporter embryos have been used to pinpoint the putative HSC precursors and the specific cellular niches in the conceptus where HSCs may initially be specified. The transcription factor Runx1 is essential for the onset of definitive hematopoiesis; lack of Runx1 abolishes HSC formation leading to embryonic lethality by E12.5 (Okuda et al., 1996; Wang et al., 1996). Runx1 is expressed in all HSCs throughout ontogeny and therefore its expression indicates putative sites of definitive hematopoiesis. In the AGM, Runx1 expression is observed both in the endothelium and subendothelial mesenchyme on the ventral side of the dorsal aorta where HSCs emerge (North et al., 1999). The Runx1 locus is also active in the homozygous Runx1-LacZ null embryos, which are unable to generate HSCs, but display LacZ expression in precursors that are attempting to generate HSCs. Interestingly, Runx1 expression can first be observed in the conceptus at E7.5 at blood islands in the yolk sac, the chorionic mesoderm and parts of the allantoic mesoderm (Fig. 4A) (Zeigler et al., 2006). By E9.5-10.5, the large chorioallantoic vessels in the placenta harbor Runx1-LacZ+ cells that are integrated within the wall of the vessel lumen, highly reminiscent of the LacZ+ cells found within the ventral wall of the dorsal aorta
A

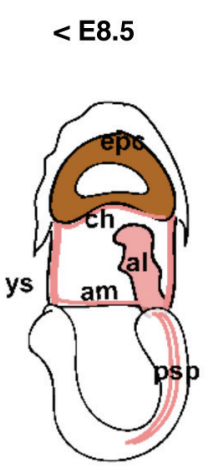

B

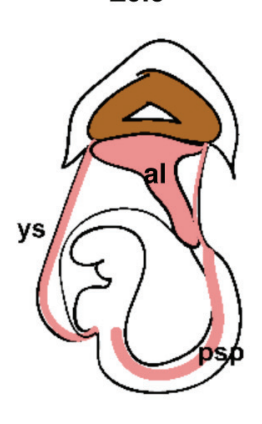

C $\quad$ E11.5

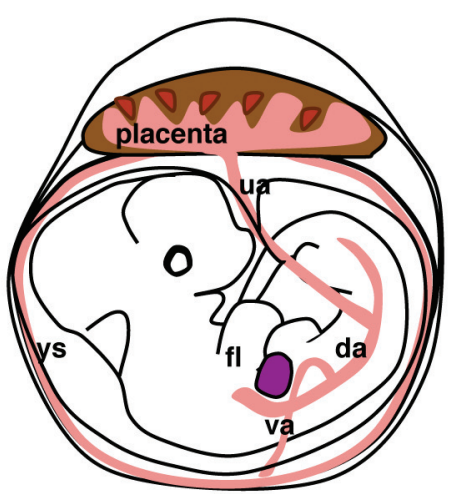

D

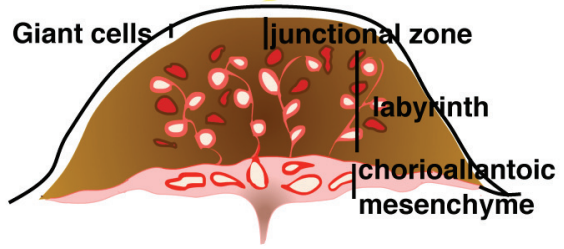

Fig. 3. Development of the mouse placenta. (A) At E7.5-8.25 the allantois (red) has formed from mesodermal precursors from the primitive streak, and is growing towards the ectoplacental cone (brown). (B) Fusion of the allantois with the chorionic mesoderm occurs at E8.5, concomitant with the onset of heartbeat. Subsequently chorioallantoic mesoderm interdigitates with the trophoblasts and the placental vasculature starts to form. (C) By E11.5 large vessels that connect to the umbilical cord have formed in the chorioallantoic mesenchyme, and the feto-placental circulation is fully established. The placenta labyrinth is still developing and is therefore an active site of vasculogenesis/angiogenesis. (D) A E12.5 cross-section of the placenta displays the different regions of the placenta, namely the chorioallantoic mesenchyme including the large vessels of the placenta (in red) and the placenta labyrinth, which is a unique region including trophoblast-lined maternal blood spaces (red spaces surrounded by brown trophoblasts) and fetal vessels lined by fetal endothelium (red vessels with lumens). al, allantois; ch, chorion; am, amnion; epc, ectoplacental cone; ys, yolk sac; psp, para-aortic splanchnopleura; da, dorsal aorta; ua, umbilical artery; va, vitelline artery; fl, fetal liver. 
(Rhodes et al., 2008) (Fig. 4B). As LacZ+ cells are found in the large vessels of the chorioallantoic mesenchyme in both the heterozygote Runx $1^{\mathrm{LacZ} /+}$ and homozygote Runx $1^{\mathrm{LacZ} / \mathrm{LacZ}}$, it was suggested that these vessels are a site of HSC origin. In comparison, as round LacZ+ definitive hematopoietic cells localized to the fetal labyrinth vessels in placentas of Runx $1^{\text {LacZ/+ }}$ embryos, but not Runx1 $1^{\text {LacZ/LacZ }}$ knockout embryos, it was suggested that the placental labyrinth vasculature might be a niche that becomes colonized by HSCs generated in the placenta and other sources (Fig. 4C) (Table 1). Furthermore, many of the round LacZ+ cells within the labyrinth vessels were mitotically active and formed small clusters, prompting the hypothesis that the placental labyrinth vasculature is a site for HSC expansion (Rhodes et al., 2008). In summary, these data suggested that the large vessels in the chorioallantoic mesenchyme may generate HSCs, similar to the large dorsal aorta and adjacent large vessels in the AGM region, while the small fetal labyrinth vessels of the placenta provide a niche in which HSCs may expand and mature. Indeed, many Runx1-LacZ+ cells in the chorioallantoic and labyrinthine blood vessels co-expressed the embryonic HSC/progenitor marker CD41 (Rhodes et al., 2008). However, in addition to being expressed in the nascent HSCs/progenitors, Runx1 is also expressed in non-hematopoietic cells. In the dorsal aorta, Runx1LacZ cells are not only found in endothelial cells, but in the mesenchyme underneath. In the placenta, oblong-shaped LacZ+ cells fill the chorioallantoic mesenchyme and may in part represent precursors for primitive macrophages that are found in the stroma (Rhodes et al., 2008). In addition, the Crypts of Duval, which are derived from ectoplacental endoderm, also were LacZpositive (Rhodes et al., 2008) (Fig. 4B). Due to the observed expression of Runx1 in other cells than definitive HSCs/progeni- tors, hematopoietic specific markers had to be used to verify the hematopoietic nature of the cells. Indeed, expression of the hematopoietic specific marker CD41 was observed in cells attached to the luminal side of the large vessels in the the placenta in both control and circulation-deficient $\mathrm{Ncx} 1^{-/-}$embryos, implying that the placental vasculature is capable for de novo hematopoiesis (Table 1, Fig. 4D, E).

\section{Hemogenic endothelium as a source of HSCs}

Definition of the precursor from which HSCs are formed has been controversial. One of the two major theories of HSC origin proposes that HSCs are generated from hemogenic endothelium, whereas an alternative theory posits that HSCs are specified in the subendothelial mesenchyme and migrate through the endothelial wall into circulation (Bertrand et al., 2005; North et al., 2002). Common ancestry for HSCs and endothelium has been proposed for a long time based on the fact that a large number of transcription factors and surface markers are shared between both lineages. All blood and endothelial cells originally develop from Flk1+ mesodermal precursor; however, Flk1+ becomes downregulated in hematopoietic cells after their emergence (Rhodes, Van Handel and Mikkola, unpublished data 2009). In contrast, the pan-endothelial marker CD31 (PECAM) remains expressed in HSCs throughout their ontogeny (Baumann et al., 2004; Taoudi and Medvinsky, 2007), while CD34 is expressed in endothelium as well as fetal HSCs, but is downregulated in the quiescent adult LTR-HSCs during early post-natal life (Ogawa et al., 2001). VE-Cadherin, another vascular marker that is a component of the endothelial cell adherens junctions, remains expressed in HSCs from their emergence through midgestation

Fig. 4. Localization of developing HSCs in the placenta. (A) $A$ crosssection of a pre-circulation Runx1-LacZ conceptus documenting Runx 1 expression in the chorionic mesoderm (black arrow) and the blood-islands of the yolk sac. The blue arrow denotes the ectoplacental cone. Image is courtesy of Dr. Nancy Speck. (B) The large vessels of the chorioallantoic mesenchyme harbor LacZ+ cells within the wall of the vessel (arrow) at E11.5, the time when HSCs emerge. The mesenchyme contains two distinct populations of $\mathrm{LacZ}+$ cells: oblong-shaped cells that straddle within the stromal cells (arrowhead), and cuboidal LacZ+/cytokeratin+ cells derived from ectoplacental endoderm that have an organized structure (asterisk). (C) LacZ+ definitive hematopoietic stem/progenitor cells localize to the fetal labyrinth vasculature. $(\mathbf{A}, \mathbf{B}, \mathbf{C})$ Runx 1 LacZ is in blue. (B,C) Laminin (pink) marks mesodermal tissues while cytokeratin (brown) marks trophoblasts

A

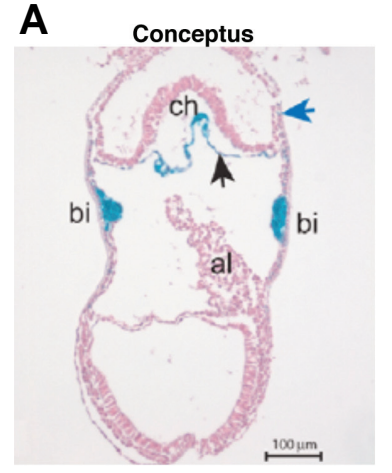

D $\quad$ wT

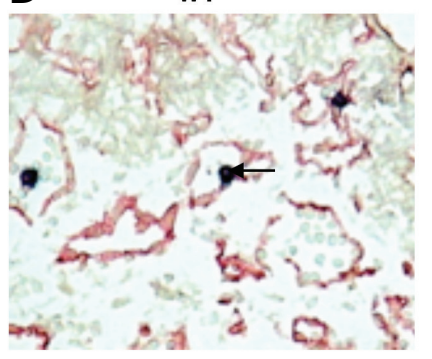

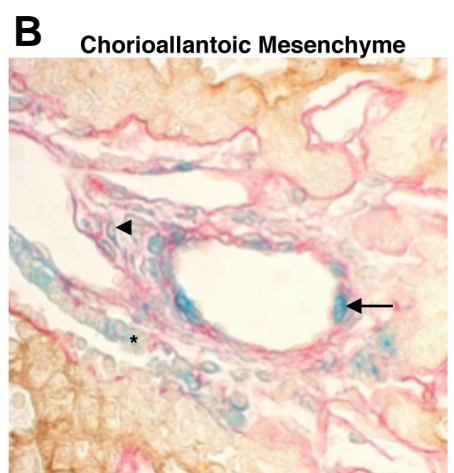

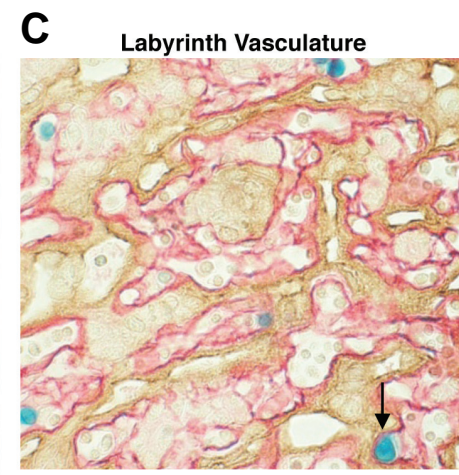

E

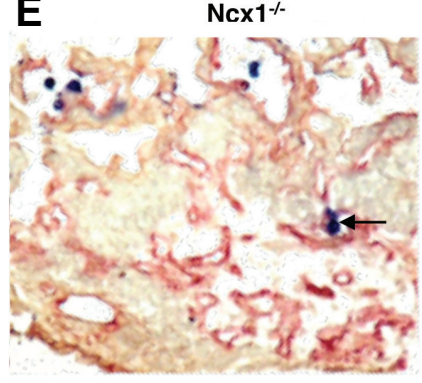

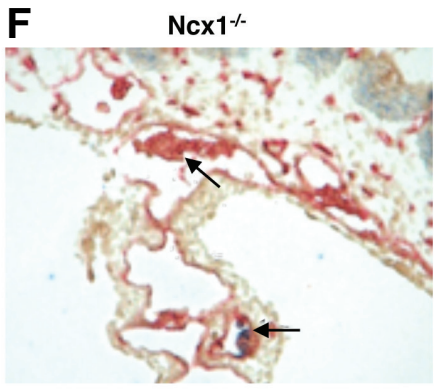

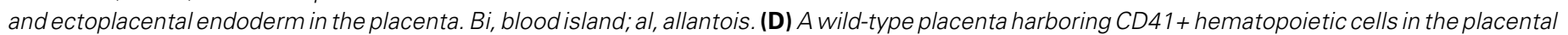

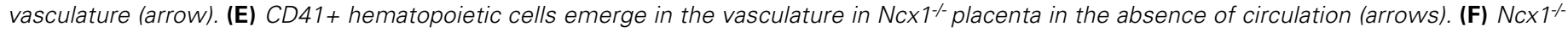

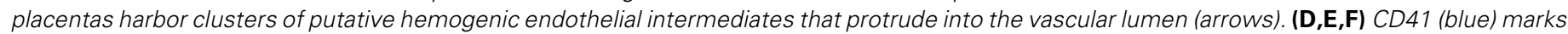
hematopoietic cells, whereas CD31 (red) identifies endothelium. Cytokeratin (brown) marks the trophoblasts. 
(Kim et al., 2005; Taoudi et al., 2005). However, the shared marker expression alone does not prove their common origin. Use of cell tracing technologies has now made it possible to verify the hypothesis that HSCs are generated from the vasculature. First, lineage tracing experiments in mice using VE-Cadherin as a marker revealed hematopoietic potential in the endothelial cells in the AGM, the placenta and the yolk sac, but not the fetal liver (Zovein et al., 2008). Furthermore, conditional deletion of Runx1 in VE-Cadherin expressing cells led to a drastic reduction of hematopoietic activity throughout the conceptus, including the AGM and the placenta (Chen et al., 2009). As VE-Cadherin is expressed in the endothelium and not in subendothelial mesenchymal cells, these results confirmed that HSCs emerge from the endothelium. The capacity of the endothelium to generate hematopoietic cells was visualized by time-lapse experiments (Eilken et al., 2009). In that study, individual ES-cell derived endothelial sheet colonies were monitored over many days and shown to lose endothelial morphology, marker expression and functional properties, while up-regulating hematopoietic markers CD41 and CD45 and acquiring hematopoietic progenitor morphology. Furthermore, our recent studies show that sorted endothelial cells from the placenta, the AGM and the yolk sac, give rise to hematopoietic progenitors when co-cultured on supportive stroma (Rhodes, Van Handel and Mikkola unpublished data 2009). Studies on the circulation-deficient $\mathrm{Ncx} 1^{-/-}$embryos further suggested that hematopoietic stem/progenitor cells develop from endothelium, as the blood vessels of these placentas harbored unique clusters of cells containing putative hemogenic endothelial intermediates that were protruding into the lumen (Fig. 4F). All together, these studies present compelling evidence that definitive hematopoietic stem- and progenitor cells arise from an endothelial precursor.

It still remains to be defined how hemogenic endothelium is established, and what makes it distinct from other non-hemogenic arterial endothelium. The study by Zovein et al. suggests that unique precursor cells in the mesenchyme underlying the dorsal aorta and other hemogenic arteries could be the precursors that give rise to hemogenic endothelium (Zovein et al., 2008). Defining these precursors that give rise to hemogenic endothelium and definitive HSCs will be required to understand the regulatory mechanisms that drive the fate decisions required for generating functional HSCs de novo.

\section{Conservation of the placenta or analogous vascular organs as sites of hematopoiesis across species}

The establishment of the hematopoietic system during embryogenesis is a highly conserved process between vertebrate species such as mouse, human and avian. In all organisms named above, HSCs emerge from the dorsal aorta in the embryo proper and other major arterial vasculature, and the same transcription factors and signaling pathways appear to direct this process (Martinez-Agosto et al., 2007). Although the placenta is unique to eutherian mammals, structures with similar mesodermal origin are also found in other species (Fig. 5).

As noted earlier, the avian allantois is a mesodermal appendage with comparable developmental origin as the mammalian allantois, and it harbors multipotential hematopoietic cells that possess bone marrow reconstitution ability (Caprioli et al., 1998; Caprioli et al., 2001). Hematopoietic potential of the avian allantois was demonstrated by a xenograft assay where the allantois of a quail embryo was isolated before the initiation of circulation to exclude cells derived from other tissues, and grafted into a chick embryo. This approach revealed that the cells derived from the quail allantois could contribute to the bone marrow of a postnatal chick, indicative of HSC activity. In addition, the presence of both hematopoietic and endothelial cells from quail origin in the chick host suggested that these cells have originated from the hemangioblast, a common progenitor of blood and endothelium, in the allantois (Fig. 5A, B).

It has also been suggested that in zebrafish the vascular plexus termed the caudal hematopoietic tissue $(\mathrm{CHT})$ or posterior blood island (PBI), which supports de novo generation, expansion and maturation of hematopoietic cells, may have similar functions in hematopoiesis as the mammalian placenta or the fetal liver (Burns and Zon, 2006; Murayama et al., 2006). Indeed, the CHT/
Fig. 5. Allantoic mesoderm and the placenta as a source of hematopoietic stemand progenitor cells across vertebrate species. (A,B) In avian embryos, hematopoietic potential was observed in the allantois (B, white arrow, Caprioli et al., 1998). (C,D) In mice, the allantois forms the mesodermal components of the placenta, including the chorioallantoic mesenchyme and its large vessels ( $D$, black arrow) and the vascular labyrinth. These regions have the ability to generate and expand hematopoietic stem or progenitor cells, respectively. (E,F) The human placenta is formed as the allantoic mesoderm invades the trophoblast-derived primary villi and forms the fetal vasculature and mesenchyme. The chorionic plate vessels and the vasculature in the villi ( $F$, black arrow) are putative hematopoietic niches. Light red, allantois and its derivatives; brown, trophoblast; dark red, maternal blood: yellow, yolk sac.
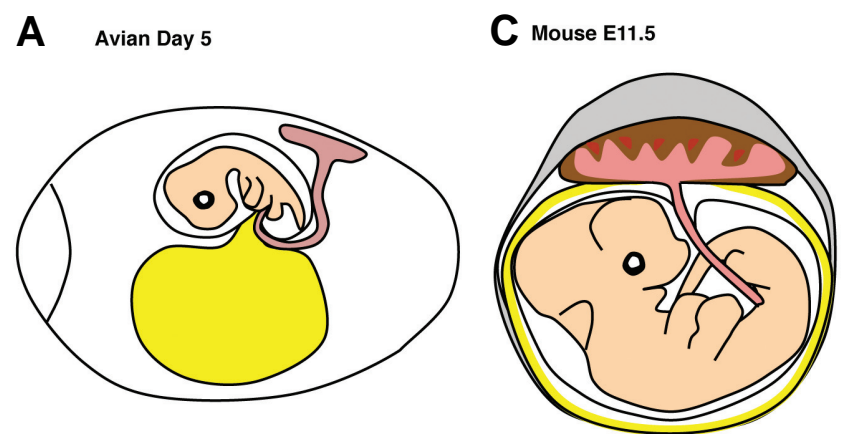

B

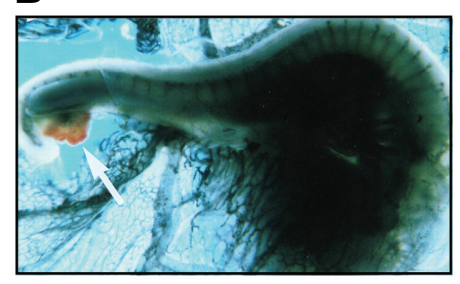

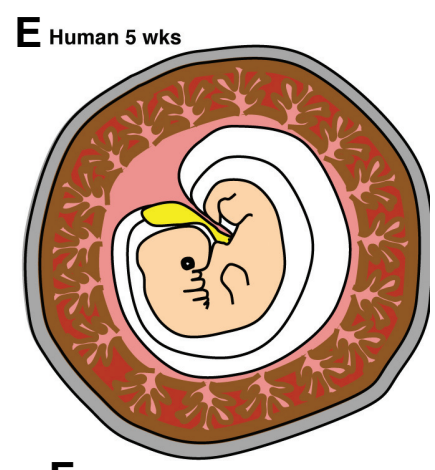

$\mathbf{F}$
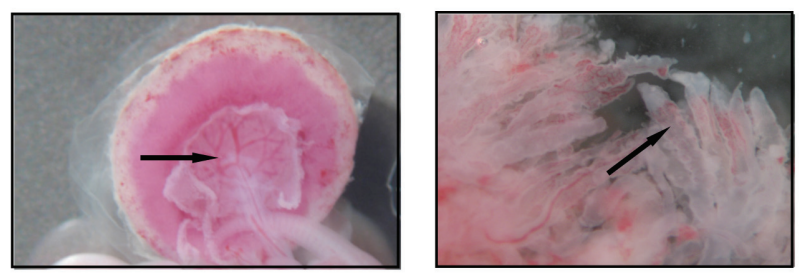
$\mathrm{PBI}$ is directly downstream of the dorsal aorta in the embryonic circulatory route, similar to the placenta in mammals. It is unknown, however, whether this vascular plexus also has exchange functions as the placenta.

Recent studies have shown that the human placenta also functions as a hematopoietic organ throughout gestation (Barcena et al., 2009a; Barcena et al., 2009b; Robin et al., 2009). Similar to the mouse placenta, the human placenta contains two main vascular structures. The chorionic plate in human is analogous to the chorioallantoic mesenchyme in mice that harbor the large blood vessels that connect to the fetus, and the villi in human placenta are analogous to the mouse placental labyrinth, which acts as the site of fetal-maternal exchange (Fig. $5 \mathrm{C}-\mathrm{F}$ ). The human placenta forms as mesoderm from the chorionic plate invades the primary villi that are comprised of trophoblast, thus giving rise to the mesodermal core that will provide the mesenchyme and vasculature of the villi. Fetal-maternal exchange takes place via the interaction between trophoblasts that sheathe the villi and maternal blood that bathe the blood lacunae. The villous ultra-structure differentiates the human placenta from the labyrinthine mouse placenta at the macroscopic level (Fig. 5 B,D); however, the placentas are remarkably similar at the cellular and molecular level (Georgiades et al., 2002) and thereby it is plausible that similar niche cells coordinate hematopoiesis in the placenta in both species. In support of this, Barcena et al. showed that the human placenta harbors progenitors with myeloerythroid potential throughout gestation and with lymphoid potential from the second trimester to birth (Barcena et al., 2009a). Importantly, the authors demonstrate an enrichment of phenotypic hematopoietic progenitors early in gestation (5-8 weeks) as compared to later gestational ages, suggesting a similar peak in hematopoietic potential in both mouse and human placentas at analogous developmental stages. Extending these findings, Robin et al. transplanted cells isolated from human placenta throughout gestation into immunocompromised mice (Robin et al., 2009). Analysis of the recipients via PCR and flow cytometry revealed the presence of engraftable human cells capable of producing lowlevel hematopoietic reconstitution as early as the first trimester and until term. Additionally, our data indicate that the human placenta may also have the capacity to generate various hematopoietic progenitor types de novo, before circulating blood cells have arrived to the placenta (Van Handel et al., 2010). Together, these findings nominate the human placenta, like the mouse placenta, as an important hematopoietic organ that may potentially generate and expand HSCs/progenitors. Thereby the placenta provides an accessible tissue source for studying the development of human HSCs and the niche cells that support this process.

\section{Conclusions and future perspectives}

Proper development and function of the placenta is essential for supporting healthy pregnancy. However, studies in recent years have revealed its vital functions are not only limited to its requirement in mediating fetal-maternal exchange, but also include a novel role as a fetal HSC niche. The uniqueness of the placenta as a hematopoietic organ relies on the findings that it is capable of de novo hematopoiesis, similar to the AGM and the yolk sac, but that it also provides a true hematopoietic niche that allows accumulation of a major HSC pool in the conceptus. Although not formally proven, the establishment of the large pool of HSCs in the placenta is likely due to the combination of at least four processes: 1) de novo HSC generation in the chorioallantoic vasculature of the placenta, 2) colonization of HSCs from the umbilical artery, the AGM and possibly yolk sac, 3) expansion of HSCs in the placental vascular labyrinth and 4) functional maturation of functionally immature "pre-HSCs" into HSCs that can engraft in standard transplantation assays. Remarkably, when HSCs reside in the placenta, they are not directed towards differentiation. These data imply that the placental microenvironment has unique properties that can support both HSC emergence and maintenance/expansion. It has been known for a long time that the placenta is rich in growth factors and cytokines that are secreted by various placental cells such as the endothelium, stromal cells, ectoplacental endoderm/Crypts of Duval, trophoblast and others. Indeed, the placenta is the largest vascular bed at this stage of development, and endothelial cells have been implicated as an important niche cell for HSCs. Furthermore, Robin et al. recently documented the presence of phenotypic pericytes surrounding vessels in the human placenta that support the ex vivoexpansion of hematopoietic stem- and progenitor cells (Robin et al., 2009). While these, and other putative niche cell types are shared with other hematopoietic organs, the trophoblasts are unique to the placenta. Their importance in supporting hematopoiesis may not be restricted to being a source of vasculogenic/angiogenic factors that are essential for forming proper placental vasculature, but may also be due to other functions that relate to the placenta's role in facilitating fetomaternal exchange. Therefore it is plausible that defects in the trophoblasts and placental vasculature may not only affect hematopoiesis in the placenta, but also in the embryo proper. On the other hand, the question has been raised how the placenta could initiate de novo hematopoiesis without the proximity of gut endoderm found in the AGM, which polarizes the dorsal aorta and induces hematopoiesis specifically on the ventral side of the aorta (Mascarenhas et al., 2009; Peeters et al., 2009; Wilkinson et al., 2009; Zon, 1995). Interestingly, the cells that form the Crypts of Duval are composed of ectoplacental endoderm, and secrete signaling molecules such as Ihh (Indian Hedgehog) that are important for hematopoietic specification from mesoderm. As they are in close proximity to the large placental vessels where HSCs emerge during midgestation, it is possible that the Crypts of Duval provide local signals required for initiating hematopoiesis in the placenta (Ogura et al., 1998; Rhodes et al., 2008).

Functional and molecular analysis of the putative niche cells in the placenta and defining the cues that dictate distinct stages of HSC development from HSC specification, emergence, and maturation through expansion will be an important task for the future. Ultimately, defining the microenvironmental cues governing HSC development may have a major impact on clinical therapeutic applications that depend on recreating the fetal hematopoietic niches for in vitro expansion of HSCs from cord blood, or de novo generation of HSCs from pluripotent cells.

\footnotetext{
Acknowledgements

H.K.A.M received funding from California Institute of Regenerative Medicine (CIRM), NIH/NHLBI and American Society of Hematology. B.V.H and K.E.R were funded by the Ruth Kirschstein National Research
} 
Service Award (GM007185), A.C by a training grant from CIRM, M.U by a fellowship from Japan Society for the Promotion of Science, and C.G by a fellowship from EMBO.

\section{References}

ALVAREZ-SILVA, M., BELO-DIABANGOUAYA, P., SALAUN, J. and DIETERLENLIEVRE, F. (2003). Mouse placenta is a major hematopoietic organ. Development 130: 5437-5444.

BARCENA, A., KAPIDZIC, M., MUENCH, M.O., GORMLEY, M., SCOTT, M.A., WEIER, J.F., FERLATTE, C. and FISHER, S.J. (2009a). The human placenta is a hematopoietic organ during the embryonic and fetal periods of development. Dev Bio/327: 24-33.

BARCENA, A., MUENCH, M.O., KAPIDZIC, M. and FISHER, S.J. (2009b). A new role for the human placenta as a hematopoietic site throughout gestation. Reprod Sci 16: 178-187.

BAUMANN, C.I., BAILEY, A.S., LI, W., FERKOWICZ, M.J., YODER, M.C. and FLEMING, W.H. (2004). PECAM-1 is expressed on hematopoietic stem cells throughout ontogeny and identifies a population of erythroid progenitors. Blood 104: 1010-1016.

BERTRAND, J.Y., GIROUX, S., GOLUB, R., KLAINE, M., JALIL, A., BOUCONTET, L., GODIN, I. and CUMANO, A. (2005). Characterization of purified intraembryonic hematopoietic stem cells as a tool to define their site of origin. Proc Nat/ Acad Sci USA 102: 134-139.

BURNS, C.E. and ZON, L.I. (2006). Homing sweet homing: odyssey of hematopoietic stem cells. Immunity 25: 859-862.

CAPRIOLI, A., JAFFREDO, T., GAUTIER, R., DUBOURG, C. and DIETERLENLIEVRE, F. (1998). Blood-borne seeding by hematopoietic and endothelial precursors from the allantois. Proc Natl Acad Sci USA 95: 1641-1646.

CAPRIOLI, A., MINKO, K., DREVON, C., EICHMANN, A., DIETERLEN-LIEVRE, F. and JAFFREDO, T. (2001). Hemangioblast commitment in the avian allantois: cellular and molecular aspects. Dev Bio/238: 64-78.

CHEN, M.J., YOKOMIZO, T., ZEIGLER, B.M., DZIERZAK, E. and SPECK, N.A. (2009). Runx 1 is required for the endothelial to haematopoietic cell transition but not thereafter. Nature 457: 887-91.

CORBEL, C., SALAUN, J., BELO-DIABANGOUAYA, P. and DIETERLEN-LIEVRE, F. (2007). Hematopoietic potential of the pre-fusion allantois. Dev Bio/301:47888.

CROSS, J.C. (2005). How to make a placenta: mechanisms of trophoblast cell differentiation in mice - a review. Placenta 26 Suppl A: S3-S9.

CUMANO, A., DIETERLEN-LIEVRE, F. and GODIN, I. (1996). Lymphoid potential, probed before circulation in mouse, is restricted to caudal intraembryonic splanchnopleura. Cel/86: 907-16.

DANCIS, J., JANSEN, V., BROWN, G.F., GORSTEIN, F. and BALIS, M.E. (1977). Treatment of hypoplastic anemia in mice with placental transplants. Blood50: 663-670.

DANCIS, J., SAMUELS, B.D. and DOUGLAS, G.W. (1962). Immunological competence of placenta. Science 136: 382-383.

DE BRUIJN, M.F., SPECK, N.A., PEETERS, M.C. and DZIERZAK, E. (2000). Definitive hematopoietic stem cells first develop within the major arterial regions of the mouse embryo. EMBO J19: 2465-2474.

DOWNS, K.M., GIFFORD, S., BLAHNIK, M. and GARDNER, R.L. (1998). Vascularization in the murine allantois occurs by vasculogenesis without accompanying erythropoiesis. Development 125: 4507-4520.

DOWNS, K.M. and HARMANN, C. (1997). Developmental potency of the murine allantois. Development 124: 2769-2780.

DUVAL, M. (1891). Le placenta des rongeurs. J Anat Physiol.

EILKEN, H.M., NISHIKAWA, S. and SCHROEDER, T. (2009). Continuous singlecell imaging of blood generation from haemogenic endothelium. Nature 457: 896-900.

GEKAS, C., DIETERLEN-LIEVRE, F., ORKIN, S.H. and MIKKOLA, H.K. (2005). The placenta is a niche for hematopoietic stem cells. Dev Cel/8: 365-375.

GEORGIADES, P., FERGUSON-SMITH, A.C. and BURTON, G.J. (2002). Comparative developmental anatomy of the murine and human definitive placentae.
Placenta 23: 3-19.

GODIN, I., GARCIA-PORRERO, J.A., DIETERLEN-LIEVRE, F. and CUMANO, A (1999). Stem cell emergence and hemopoietic activity are incompatible in mouse intraembryonic sites. J Exp Med 190: 43-52.

KIM, I., YILMAZ, O.H. and MORRISON, S.J. (2005). CD144 (VE-cadherin) is transiently expressed by fetal liver hematopoietic stem cells. Blood 106: 903905.

KOUSHIK, S.V., WANG, J., ROGERS, R., MOSKOPHIDIS, D., LAMBERT, N.A., CREAZZO, T.L. and CONWAY, S.J. (2001). Targeted inactivation of the sodium-calcium exchanger (Ncx1) results in the lack of a heartbeat and abnormal myofibrillar organization. FASEB J15: 1209-1211.

KUMARAVELU, P., HOOK, L., MORRISON, A.M., URE, J., ZHAO, S., ZUYEV, S., ANSELL, J. and MEDVINSKY, A. (2002). Quantitative developmental anatomy of definitive haematopoietic stem cells/long-term repopulating units ( $\mathrm{HSC} /$ RUs): role of the aorta-gonad-mesonephros (AGM) region and the yolk sac in colonisation of the mouse embryonic liver. Development 129: 4891-4899.

LUX, C.T., YOSHIMOTO, M., MCGRATH, K., CONWAY, S.J., PALIS, J. and YODER, M.C. (2008). All primitive and definitive hematopoietic progenitor cells emerging prior to $\mathrm{E} 10$ in the mouse embryo are products of the yolk sac. Blood 111: 3435-3438

MARTINEZ-AGOSTO, J.A., MIKKOLA, H.K., HARTENSTEIN, V. and BANERJEE, $U$. (2007). The hematopoietic stem cell and its niche: a comparative view. Genes Dev21: 3044-3060.

MASCARENHAS, M.I., PARKER, A., DZIERZAK, E. and OTTERSBACH, K. (2009). Identification of novel regulators of hematopoietic stem cell development through refinement of stem cell localization and expression profiling Blood 114: 4645-4653.

MEDVINSKY, A. and DZIERZAK, E. (1996). Definitive hematopoiesis is autonomously initiated by the AGM region. Cel/86: 897-906.

MELCHERS, F. (1979). Murine embryonic B lymphocyte development in the placenta. Nature 277: 219-221.

MIKKOLA, H.K. and ORKIN, S.H. (2006). The journey of developing hematopoietic stem cells. Development 133: 3733-3744

MULLER, A.M., MEDVINSKY, A., STROUBOULIS, J., GROSVELD, F. and DZIERZAK, E. (1994). Development of hematopoietic stem cell activity in the mouse embryo. Immunity 1: 291-301.

MURAYAMA, E., KISSA, K., ZAPATA, A., MORDELET, E., BRIOLAT, V., LIN, H.F. HANDIN, R.I. and HERBOMEL, P. (2006). Tracing hematopoietic precursor migration to successive hematopoietic organs during zebrafish development. Immunity 25: 963-975.

NORTH, T., GU, T.L., STACY, T., WANG, Q., HOWARD, L., BINDER, M., MARINPADILLA, M. and SPECK, N.A. (1999). Cbfa2 is required for the formation of intra-aortic hematopoietic clusters. Development 126: 2563-2575.

NORTH, T.E., DE BRUIJN, M.F., STACY, T., TALEBIAN, L., LIND, E., ROBIN, C., BINDER, M., DZIERZAK, E. and SPECK, N.A. (2002). Runx1 expression marks long-term repopulating hematopoietic stem cells in the midgestation mouse embryo. Immunity 16: 661-672.

OGAWA, M., TAJIMA, F., ITO, T., SATO, T., LAVER, J.H. and DEGUCHI, T. (2001) CD34 expression by murine hematopoietic stem cells. Developmental changes and kinetic alterations. Ann N Y Acad Sci938: 139-145.

OGURA, Y., TAKAKURA, N., YOSHIDA, H. and NISHIKAWA, S.I. (1998). Essential role of platelet-derived growth factor receptor alpha in the development of the intraplacental yolk sac/sinus of Duval in mouse placenta. Bio/Reprod58: 65-72.

OKUDA, T., VAN DEURSEN, J., HIEBERT, S.W., GROSVELD, G. and DOWNING, J.R. (1996). AML1, the target of multiple chromosomal translocations in human leukemia, is essential for normal fetal liver hematopoiesis. Ce//84: 321-330.

OTTERSBACH, K. and DZIERZAK, E. (2005). The murine placenta contains hematopoietic stem cells within the vascular labyrinth region. Dev Cel/8: 377387.

PALIS, J., ROBERTSON, S., KENNEDY, M., WALL, C. and KELLER, G. (1999). Development of erythroid and myeloid progenitors in the yolk sac and embryo proper of the mouse. Development 126: 5073-5084

PEETERS, M., OTTERSBACH, K., BOLLEROT, K., ORELIO, C., DE BRUIJN, M. WIJGERDE, M. and DZIERZAK, E. (2009). Ventral embryonic tissues and Hedgehog proteins induce early AGM hematopoietic stem cell development. Development 136: 2613-2621. 
RHODES, K.E., GEKAS, C., WANG, Y., LUX, C.T., FRANCIS, C.S., CHAN, D.N., CONWAY, S., ORKIN, S.H., YODER, M.C. and MIKKOLA, H.K. (2008). The emergence of hematopoietic stem cells is initiated in the placental vasculature in the absence of circulation. Cel/ Stem Cel/2: 252-263.

ROBIN, C., BOLLEROT, K., MENDES, S., HAAK, E., CRISAN, M., CERISOLI, F., LAUW, I., KAIMAKIS, P., JORNA, R., VERMEULEN, M. et al. (2009). Human placenta is a potent hematopoietic niche containing hematopoietic stem and progenitor cells throughout development. Cel/ Stem Cel/5: 385-395.

ROSSANT, J. and CROSS, J.C. (2001). Placental development: lessons from mouse mutants. Nat Rev Genet 2: 538-548.

TAOUDI, S. and MEDVINSKY, A. (2007). Functional identification of the hematopoietic stem cell niche in the ventral domain of the embryonic dorsal aorta. Proc Natl Acad Sci USA 104: 9399-9403.

TAOUDI, S., MORRISON, A.M., INOUE, H., GRIBI, R., URE, J. and MEDVINSKY, A. (2005). Progressive divergence of definitive haematopoietic stem cells from the endothelial compartment does not depend on contact with the foetal liver. Development 132: 4179-4191.

TILL, J.E. and MCCULLOCH, E.A. (1961). A direct measurement of the radiation sensitivity of normal mouse bone marrow cells. Radiat Res 14: 213-222.

VAN HANDEL, B., PRASHAD S.L., HASSANZADEH-KIABI N., HUANG A.,
MAGNUSSON M., ATANASSOVA B., CHEN A., HAMALAINEN E.I. and MIKKOLA H.K.A. (2010). The first trimester human placenta is a site for terminal maturation of primitive erythroid cells. Blood First Edition Paper, prepublished online July 13, 2010.

WANG, Q., STACY, T., BINDER, M., MARIN-PADILLA, M., SHARPE, A.H. and SPECK, N.A. (1996). Disruption of the Cbfa2 gene causes necrosis and hemorrhaging in the central nervous system and blocks definitive hematopoiesis. Proc Nat/ Acad Sci USA 93: 3444-3449.

WILKINSON, R.N., POUGET, C., GERING, M., RUSSELL, A.J., DAVIES, S.G. KIMELMAN, D. and PATIENT, R. (2009). Hedgehog and Bmp polarize hematopoietic stem cell emergence in the zebrafish dorsal aorta. Dev Cel/16: 909-916.

ZEIGLER, B.M., SUGIYAMA, D., CHEN, M., GUO, Y., DOWNS, K.M. and SPECK N.A. (2006). The allantois and chorion, when isolated before circulation or chorio-allantoic fusion, have hematopoietic potential. Development 133: 4183 4192.

ZON, L.I. (1995). Developmental biology of hematopoiesis. Blood 86: 2876-2891.

ZOVEIN, A.C., HOFMANN, J.J., LYNCH, M., FRENCH, W.J., TURLO, K.A., YANG Y., BECKER, M.S., ZANETTA, L., DEJANA, E., GASSON, J.C. et al. (2008). Fate tracing reveals the endothelial origin of hematopoietic stem cells. Cell Stem Cel/3: 625-636.

\section{Further Related Reading, published previously in the Int. J. Dev. Biol.}

See our recent Special Issue Placenta edited by Joan S. Hunt and Kent L. Thornburg at: http://www.ijdb.ehu.es/web/contents. php?vol=54\&issue=2-3

An activating mutation in the PDGF receptor-beta causes abnormal morphology in the mouse placenta

Camilla Looman, Tong Sun, Yang Yu, Agata Zieba, Aive Ahgren, Ricardo Feinstein, Henrik Forsberg, Carina Hellberg, Carl-Henrik Heldin, Xiao-Qun Zhang, Karin Forsberg-Nilsson, Nelson Khoo, Reinald Fundele and Rainer Heuchel

Int. J. Dev. Biol. (2007) 51: 361-370

Of birds and mice: hematopoietic stem cell development

Isabelle Godin and Ana Cumano

Int. J. Dev. Biol. (2005) 49: 251-257

Tracing the hemangioblast during embryogenesis: developmental relationships between endothelial and hematopoietic cells

Thierry Jaffredo, Karine Bollerot, Daisuke Sugiyama, Rodolphe Gautier and Cécile Drevon Int. J. Dev. Biol. (2005) 49: 269-277

\section{Commitment of hematopoietic stem cells in avian and mammalian embryos: an ongoing} story

Françoise Dieterlen-Lièvre

Int. J. Dev. Biol. (2005) 49: 125-130

\section{Embryonic development of the human hematopoietic system}

Manuela Tavian and Bruno Péault

Int. J. Dev. Biol. (2005) 49: 243-250

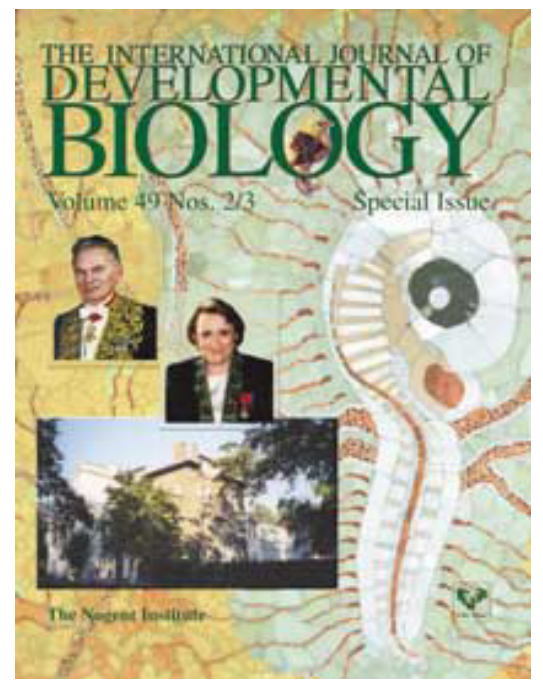

5 yr ISI Impact Factor $(2009)=3.253$

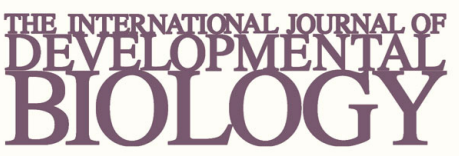

Volume 54 Nos. $6 / 7$ Special Issue

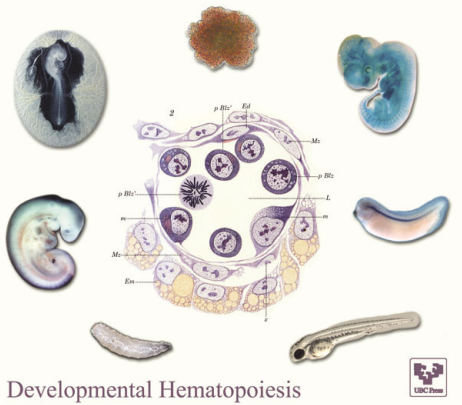

